\title{
Study of Burning Characteristics of Porous Combustibles Soaked With a Liquid Oxidizer
}

\author{
Daiki Matsugi ${ }^{1}$, Yuji Nakamura ${ }^{1}$ \\ ${ }^{1}$ Department of Mechanical Engineering, Toyohashi University of Technology \\ 1-1 Hibarigaoka, Tempaku, Toyohashi, Aichi, Japan \\ matsugi.daiki.bl@tut.jp; yuji@me.tut.ac.jp
}

\section{Extended Abstract}

With an increasing awareness of environmental concerns (e.g., ozone depletion, acid rain, etc.), "green" propellants replacing into AP-based (ammonium perchlorate, chlorin-origin gunpowder) solid propellants have been of a great interest for sustainable developments [1][2]. In order to reach this goal, explosive, yet stable burning with low-harmful fuel and oxidizer combination is highly demanded. Employing a porous combustible well-soaked with a liquid oxidizer, featuring large specific surface-volume ratio $(\mathrm{S} / \mathrm{V})$, is one of promising methodologies. Nagata et al. [3] investigated pressure dependency of the burning rates of porous polymer with liquid oxygen (LOX) in nitrogen environment and successfully obtained a maximum burning rate at the rate of $20 \mathrm{~mm} / \mathrm{s}$ at $0.3 \mathrm{MPa}$ in absolute. It is successfully noticed that the measured burning rate corresponds to two-times higher than the AP-based propellant [4]. This unique idea proves that the "well-mixing" specimen can be an alternative candidate for the AP-based propellant, however, detail of flame structure cannot be provided due to the system limitation. Indeed, large data scattering of the burning rates was frequently identified which lose the reproducibility. In order to go detail to discuss about the burning characters with theory basis, whole experimental system shall be upgraded to deliver the comparable data against the theoretical study.

In this work, the tested specimen is replaced as follows; a polyethylene foam having 0.7 fuel porosity, as a porous combustible, soaked with $80 \mathrm{wt} \%$ hydrogen peroxide (not LOX), as the liquid oxidizer, for ease to handle in room temperature. We then newly introduced a large volume chamber which a quartz window is equipped with, to allow the direct observation during the burning event. Experiment is carried out at an atmospheric environment ( $0.1 \mathrm{MPa}$ in absolute, $21 \%$ oxygen concentration in vol.). After an ignition at top surface of the specimen with a U-shaped nickel-chrome wire, a successive-burning event is followed. The burning events are recorded by a video camera through the quartz window, and the burning rates are measured by carefully tracking a location of burning surface with an image processing software.

The direct observation reveals that two distinctive flames are observed; one is a premixed-like surface flame exposed onto the burning surface, the other is an elongated diffusion-like flame reacted with ambient air, and an 1-D burning behaviour at which the top surface recedes are clearly identified. Careful measurement of the burning rates with the image processing shows that the steady burning mode at the rate of $1 \mathrm{~mm} / \mathrm{s}$ is successfully obtained under the condition studied. This work ensures that there is 1-D steady state solution in this system which might be possible to study theoretically. Currently we are working on theoretical model to predict the burning character, including the achievable pressure dependence on burning rate, which is currently only available through the corresponding measurement.

\section{References}

[1] N. Wingborg, M. Skarstind, M. Sjöblom, A. Lindborg, M. Brantlind, J. Johansson, S. Ek, M. Liljedahl and J. Kjellberg , "GRAIL: Green solid propellants for launchers," in Proceedings of the 7th European Conference for Aeronautics and Space Sciences, Milan, Italy, 2017.

[2] D. Pastrone, "Approaches to low fuel regression rate in hybrid rocket engines," Int. J. Aerospace Eng., vol $2012,2012$.

[3] H. Nagata, R. Akiba, N. Tanatsugu, M. Kohno, R. Yokota and S. Kayuta, "A preliminary study of the wet towel hybrid rocket motor," Aeronaut. and Spa. Sci. Jpn, vol. 45, pp. 365-370, 1997.

[4] G. P. Sutton, O. Biblarz, Rocket Propulsion Elements 9th edition, Wiley, 2017, pp. 446-496. 